\title{
A Design Method of Low-frequency Power Transmitter based on OPA549
}

\author{
Linyu Wang ${ }^{a}$, Junxian Meng ${ }^{\mathrm{b}}$ \\ College of Information and Communication Engineering of Harbin Engineering University, Harbin \\ 150001, China \\ awanglinyu@hrbeu.edu.cn, bjunxianmeng@hrbeu.edu.cn
}

Keywords: Communication system; Power transmitter; OPA549; Low frequency.

\begin{abstract}
We can interpret communication system as a system which transmit and receive signals. Transmitters play an important role in this system. In order to make sure that receivers can receive the signal, we should offer enough power transmitted by transmitters. Besides, we should also guarantee the stability and reliability. This text introduces a low-frequency power transmitter based on OPA549, and went on a series of experiments both in laboratory and field test, and receive the desired effect.
\end{abstract}

\section{Introduction}

Recently, although we go through a huge change where high-frequency circuits spread quickly, however, low-frequency signals still have advantages. Though high-frequency signals are less interfered and have shorter wavelength when compared to low-frequency signals, low-frequency circuits own special advantages. It makes it a little easier to design low-frequency circuits, for we do not need consider something about transmission line, and it is easier when considering DSP, besides, when transmitting signals in the medium, we can get more signal power and less attenuation comparing to high-frequency. So, low-frequency signals are widely applied in many fields. Therefore this time we design power transmitter with low-frequency.

\section{Hardware Design}

Normally, no matter whether we transmit analogy signals directly or processed by D/A converters, the signals share no power, and cannot be transmitted through antenna. So we should amplify its power using power amplifier.

Because the signal we transmit has a certain amplitude, and its value is very small, between $5 \mathrm{mv}$ to $50 \mathrm{mv}$ usually, accordingly, we should amplify its value when we begin to amplify its power. In order to achieve desired result, we choose two-stage amplifiers before transmit signal power. First pga206 and pga103, and then opa211. The pga206 are digitally programmable gain instrumentation amplifiers that are ideally suited for data acquisition systems. Gains are selected by two CMOS/TTL -compatible address lines. Its digital gains are 1, 2, 4, and 8 . We can get wide range of programmable gains from 1 to 1000 by combing pga206 with pga103. See figure 1 . We use the form of two-stage cascade connections to realize this result. The total gains is the product of pga206 and pga103. See Table 1.

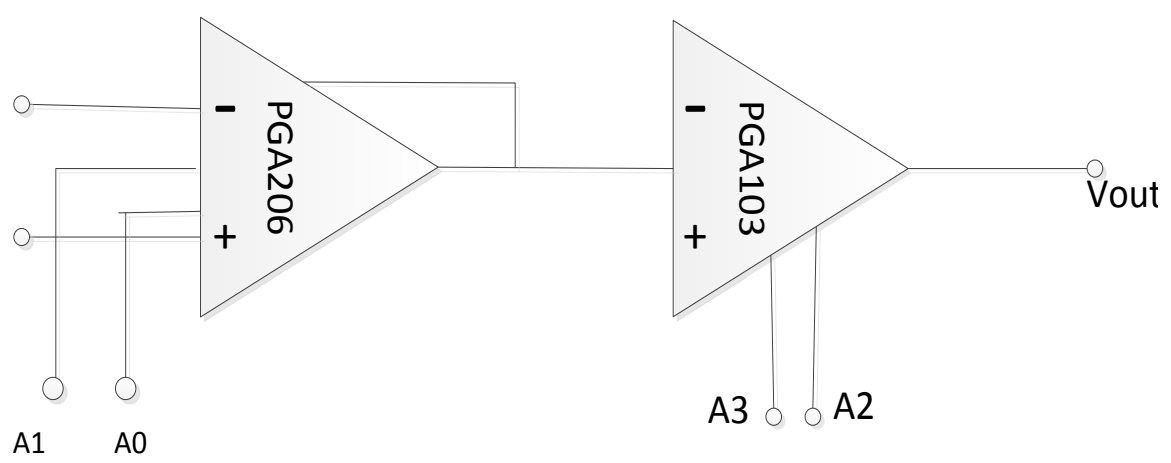

Fig. 1: The circuit of pga206 and pga103 
Table 1: Gain magnitude

\begin{tabular}{c|c|c|c|c}
\hline Gain(V/V) & A1 & A0 & A3 & A2 \\
\hline 1 & 0 & 0 & 0 & 0 \\
\hline 2 & 0 & 1 & 0 & 0 \\
\hline 5 & 1 & 0 & 0 & 0 \\
\hline 10 & 1 & 1 & 0 & 0 \\
\hline 20 & 0 & 1 & 0 & 1 \\
\hline 50 & 1 & 0 & 0 & 1 \\
\hline 100 & 1 & 1 & 0 & 1 \\
\hline 200 & 0 & 1 & 1 & 0 \\
\hline 500 & 1 & 0 & 1 & 0 \\
\hline 1000 & 1 & 1 & 1 & 0 \\
\hline
\end{tabular}

Known by the table 1, we know that we can get desired gains by controlling every electrical level of both pga206 and pga103.

The final circuit is as following:

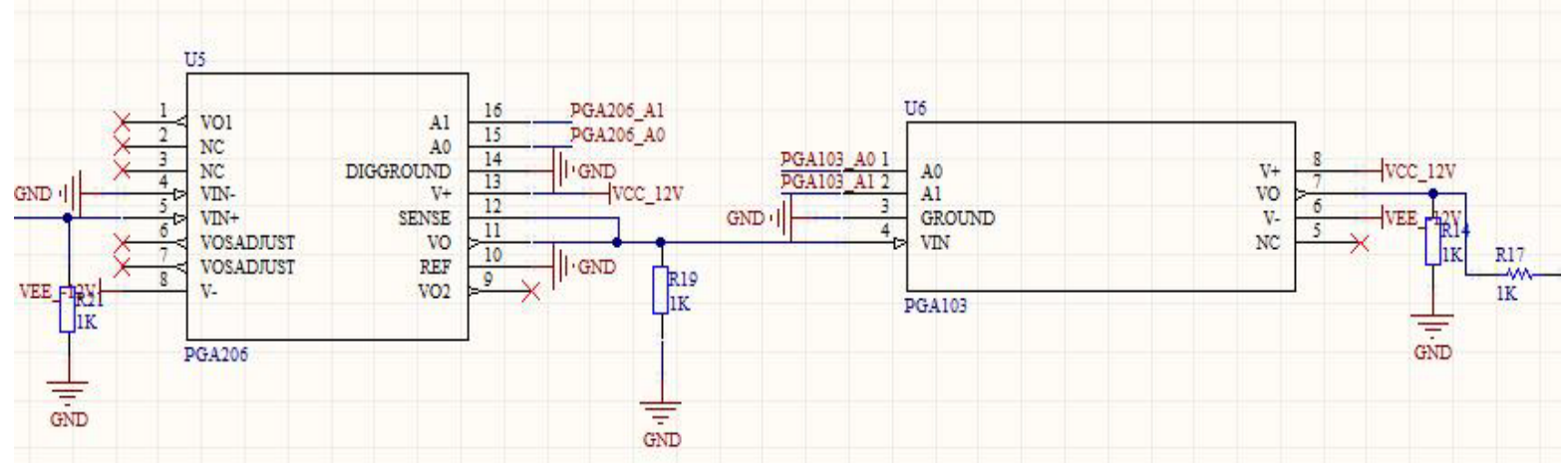

Fig. 2: Final circuit of pga 206 and pga103

The level of A0, A1, A2, and A3 are due to chip TLP521-4, a kind of programmable controller. And then signal goes two stage-amplifier. We adopt the chip opa211 offered by TI Corporation. It features low power, low noise and high precision, its voltage noise is only 1.1nV/sqr (Hz). (Actually, as this circuit is very common in other analogy circuits books, so this paper neglect the circuit).

Actually, we can change the gain amplifier by regulating the value of feedback resistance throughThis circuit.

When designing the circuit, we can add a capacitor which can prevent self-excitation. The value of the capacitor should not be in the order of pF, otherwise it cannot work normally.

Finally, our work is to design PA.

Given the premise that voltage are determined, PA is likely to output power as high as possible. So when begin to design the circuit, we should distinguish it from small signal amplifiers. From the point of control and energy conversion, there is no distinctive difference between traditional amplifier and PA, but traditional amplifier aims at amplifying its voltage or current.

The important goals of PA are maximum output power and conversion efficiency. This time we use a new chip offered by TI which can output high voltage and high current called opa549, it's a low-cost, high-voltage/high-current operational amplifier ideal for driving a wide variety of loads. It's mainly features are: high output current 8A continuous and 10A peak; Wide power-supply range, single supply: $+8 \mathrm{~V}$ to $+60 \mathrm{~V}$, dual supply: $\pm 4 \mathrm{~V}$ to $\pm 30 \mathrm{~V}$; Wide output voltage swing; Thermal shutdown protected. Since the signal is a differential signal in the propagation medium, the signal should be transmitted in two channels. One is non-inverting input and the other is inverting input. In 
order to increase output current, we use two chips opa549 in every channel. As the output current and power are very high, we also should consider thermal management. See figure 3.
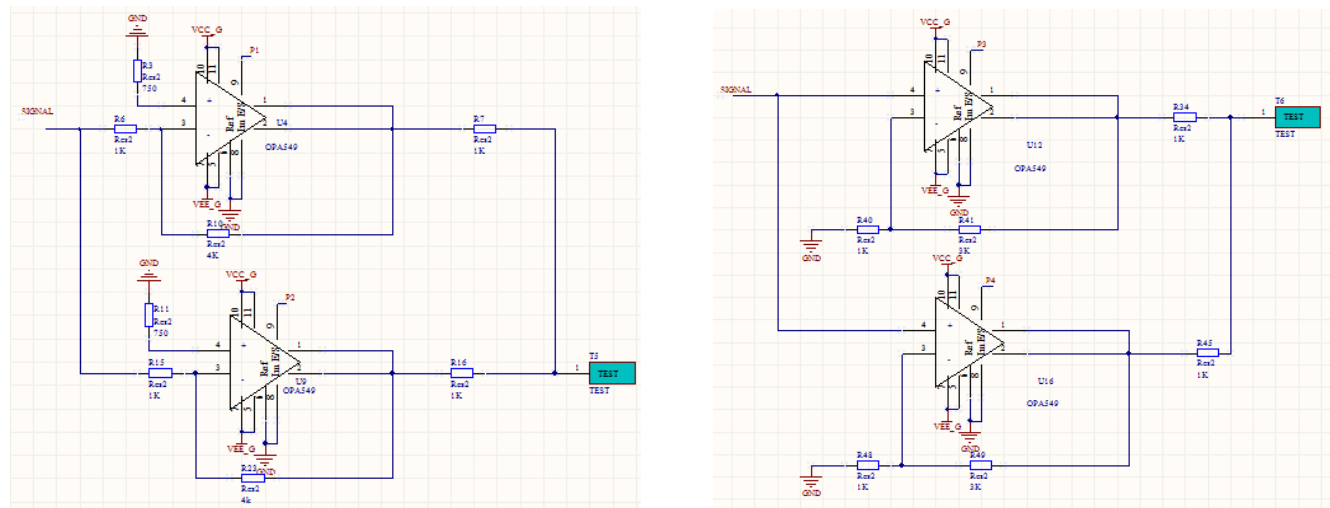

Fig. 3: Non-inverting and inverting circuit

As the output current is very high, the circuit should be protected using indicator light or buzzer in case of failure. In order to make result easier to be observed, we use buzzer. See figure 4.

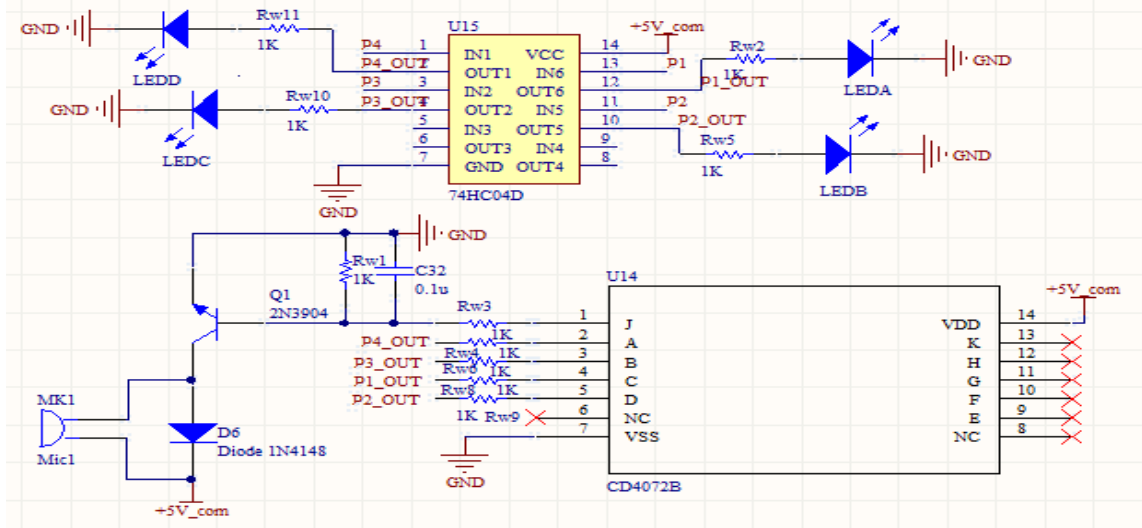

Fig. 4: Protection circuit

\section{Result}

According to the design thought talked above, the final real product photo is as following:

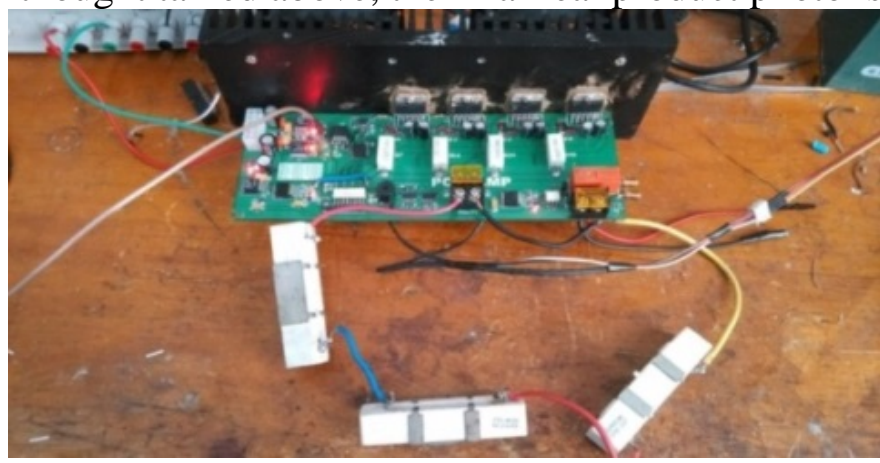

Fig. 5: Real product photo of PA

When testing, we connect $3 \Omega$ resister with PA to receive impedance matching. Add sinusoidal Signal with signal source, and change the value of variable resistance so as to change output power.

Observe the result with oscilloscope. See figure 6. 


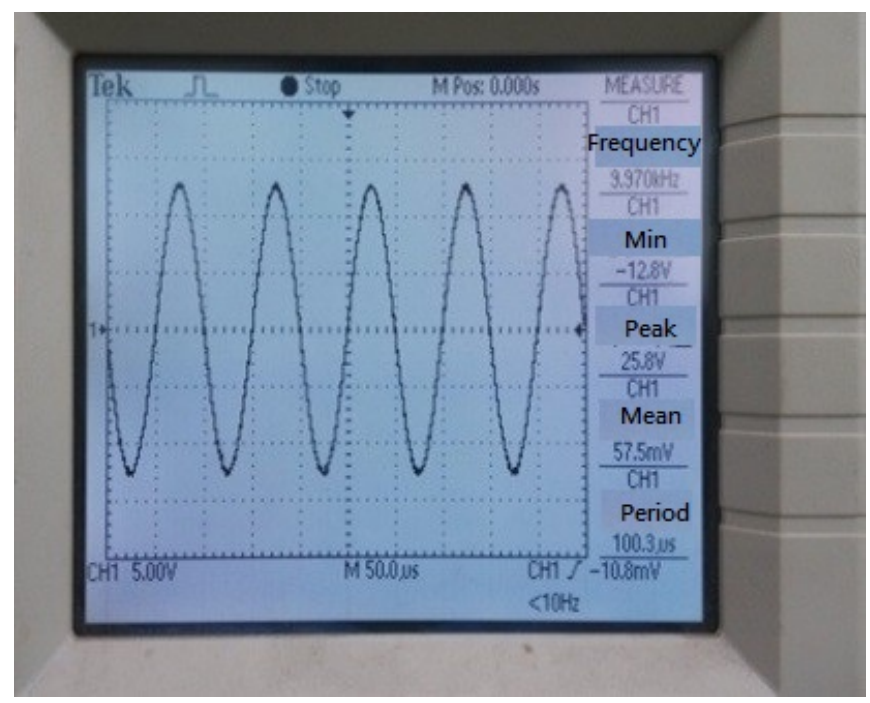

Fig. 6: Simulate output waveform

We can compute the output power according to the output voltage shown above:

$(25.8 / \sqrt{2})^{2} \times 1 / 3=110.94(W)$

According to real field test, it can meet our requirements on the order of this power.

\section{Summary}

According to practical design and test, this context explores the feasibility of low-frequency transmitter based on chip opa549. And the result proves that this transmitter can reach design requirements. This system proves to be stable and is easily to be implemented, so it has a wide-ranging applied backgrounds.

\section{Acknowledgment}

This paper is funded by the International Exchange Program of Harbin Engineering University for Innovation-oriented Talents Cultivation, International Science \& Technology Cooperation Program of China (2014 DFR10240), National Natural Science Foundation of China (612111070), China Postdoctoral Science Foundation (2013T60346), Harbin science and technology research projects (2013AE1BE003), Heilongjiang province natural science foundation projects (F201344).

\section{References}

[1] Donald A. Neamen. Eletronic Circuit Analysis and Design. BeiJing: Tsinghua University Press, 2009.

[2] Texas Instruments Incorporated. -36V, -200mA, Ultralow-Noise, Negative Linear Regulator. 2012

[3] Principles of Communications. BeiJing: National Defense Industry Press,2012.

[4] Momma H, Tsuchiya T. Underwater communication by electric current. OCEANS'76, 1976: 24C-1 - 24C-6.

[5] The Research of Underground Short Distance Visible Light Communication Technology. Shandong: Ocean University of China.

[6] Marc T.Thompson. Practical Analog Circuit Design[M]. Beijing: Posts \& Telecom Press.

[7] Momma H, Tsuchiya T. Underwater communication by electric current. OCEANS'76, 1976: 24C-1 - 24C-6. 